\title{
Rethinking AI magazine again
}

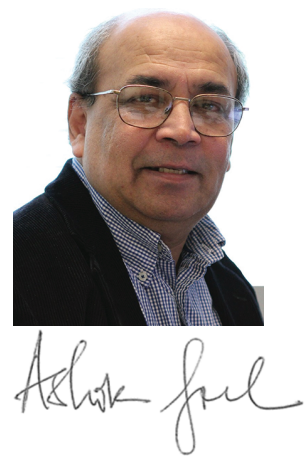

After one year as associate editor (August 2015 - August 2016) and four and half years as editorin-chief of AI Magazine (August 2016 - February 2021), I am stepping aside. Fortunately, I will continue my association with AIM as editor-in-chied emeritus. By 2015, the current boom in interest in AI already had begun (though it was less perspicuous then than it is now) and thus it was a good time to rethink AI Magazine. First, given that AI was attracting increasingly large number of scholars, I thought that AIM could have a larger number of columns that reflected more diverse perspectives. Second, given that AI was playing an increasingly more prominent role in society, I thought that AIM could become a more important forum for discussions about AI and society. Third, given that we live in the age of social media, I thought that AIM could have a stronger online presence. I am pleased that over the last 5 years, AI Magazine has made significant progress along these three themes of participation, content, and medium, respectively.

Thus, AI Magazine now has multiple new columns, for example, Odd Erik Gundersen's column on Reproducible $A I$ and Christopher Welty's column on AI Bookie in which two or more AI scholars make bets about the future of AI. Also, most issues of AIM since Winter 2017 have had at least one article relating to AI and Society; the Winter 2020 issue specifically emphasized AI for Social Impact and similarly the Spring 2021 explicitly focused on AI Social Disruption. In addition, in June 2020, we launched Interactive AI Magazine (InteractiveAIMag.org) that not only provides a gateway to articles published in AIM, but also gives access to news and videos related to AAAI as well as articles pulled from AI Topics (AITopics.org). If you, gentle reader, have not yet visited Interactive $A I$ Magazine, I recommend you do so.

At the same time, we continued to build on the extant strength of AI Magazine. As just one example, the Summer 2020 special issue on DARPA's Impact on AI contains excellent review articles on five core topics in AI:
Knowledge Representation and Reasoning, Human Language Technology, Machine Learning, Vision and Robotics, and Integrated AI Systems. These review articles have been written by some of the leaders in AI research on these topics.

Of course all this made for a lot of work. Given the boom in interest in AI, the number of submissionsabstracts, reports, columns, videos, news items, and full articles-received by AI Magazine and Interactive AI Magazine together now is several times the number in 2016. Further, the turnaround time for Interactive AI Magazine is much shorter than for AI Magazine. I am deeply grateful to an excellent editorial team for making this redesign and expansion of AI Magazine successful. At different points over the last 5 years, this team has included Odd Erik Gundersen, Robert Morris, Hector Munoz-Avila, Pearl Pu, Sandip Sen, and Kristen Brent Venable as Associate Editors; Pushpak Bhattacharyya, Bistra Dilkina, Odd Erik Gundersen, James Spohrer, Christopher Welty, and Michael Wollowski as columnists; Marie des Jardins, Kenneth Forbus, Kenneth Ford, Sven Koenig, David Leake, and Ramon Lopez de Mantaras, Sheila McIlraith, and Qiang Yang as members of the Advisory Board; Joshua Ecroth as the liaison to AI Topics; Kapil Patnaik of Caretecher as the developer of Interactive AI Magazine's infrastructure; and Ida Camacho as the editorial assistant.

I am also very grateful for the support of Yolanda Gil, Past President of AAAI: it is during her tenure that AAAI first provided funding to hire Ida Camacho as an editorial assistant for AI Magazine well as to develop and launch Interactive AI Magazine. In addition, I am grateful to Bruce Buchanan and Reid Smith of i2k Connect for their support for linking Interactive AI Magazine and AI Topics.

However, the work on AI Magazine and Interactive AI Magazine as envisioned in Rethinking AI Magazine 5 years back is incomplete. To fully realize the vision, AIM needs an even larger number of columns representing even more diverse perspectives, more of its contents needs to be related to AI and society, and the Interactive AI Magazine needs to become, well, more interactive.

Nevertheless, AI Magazine and Interactive AI Magazine are in a strong position going forward. As just one example, 
even as I write this article in early February of 2021, themes and articles for the rest of the calendar year already are lined up: articles based on IAAAI 2020 for Summer 2021, articles on Recommender Systems for Fall 2021, and articles describing the NSF Convergence Accelerator Program for Winter 2021; we also have a strong proposal for a special issue in Spring 2022.

We have formed a strong editorial team to take $A I$ Magazine and Interactive AI Magazine to new levels of excellence. Moving forward, Odd Erik Gundersen and Kristen Brent Venable will be the new Co-Editors-in-Chief, and Joshua Ecroth, Robert Morris, Pearl Pu, Sandip Sen, and Michael Wollowski will be the Associate Editors. The recently reconstituted, international and distinguished Advisory Board includes David Aha, Henrik Christensen, James Hendler, Sarit Kraus, Melanie Mitchell, Jordan Pollack, Brian Scassellati, Elizabeth Sonenberg, and Zhi-hua Zhou. I sincerely thank all of them for their service and wish them success.

Finally, I thank AAAI for giving me the opportunity to lead AI Magazine and launch Interactive AI Magazine. This has been an exciting time for AI and AAAI, for
AI Magazine and Interactive AI Magazine, as well as for me.

Ashok Goel

Georgia Institute of Technology, Atlanta, Georgia, USA

\section{Correspondence}

Ashok Goel, Georgia Institute of Technology, Atlanta, GA 30332, USA.

Email: ashok.goel@cc.gatech.edu

\section{AUTHOR BIOGRAPHY}

Ashok Goel is a professor of computer science and human-centered computing in the School of Interactive Computing at Georgia Institute of Technology in Atlanta, Georgia. He was editor-in-chief of AI Magazine from 2016 to 2021 and founding editor of Interactive AI Magazine in 2020. He is now AIM's editor-inchief emeritus.

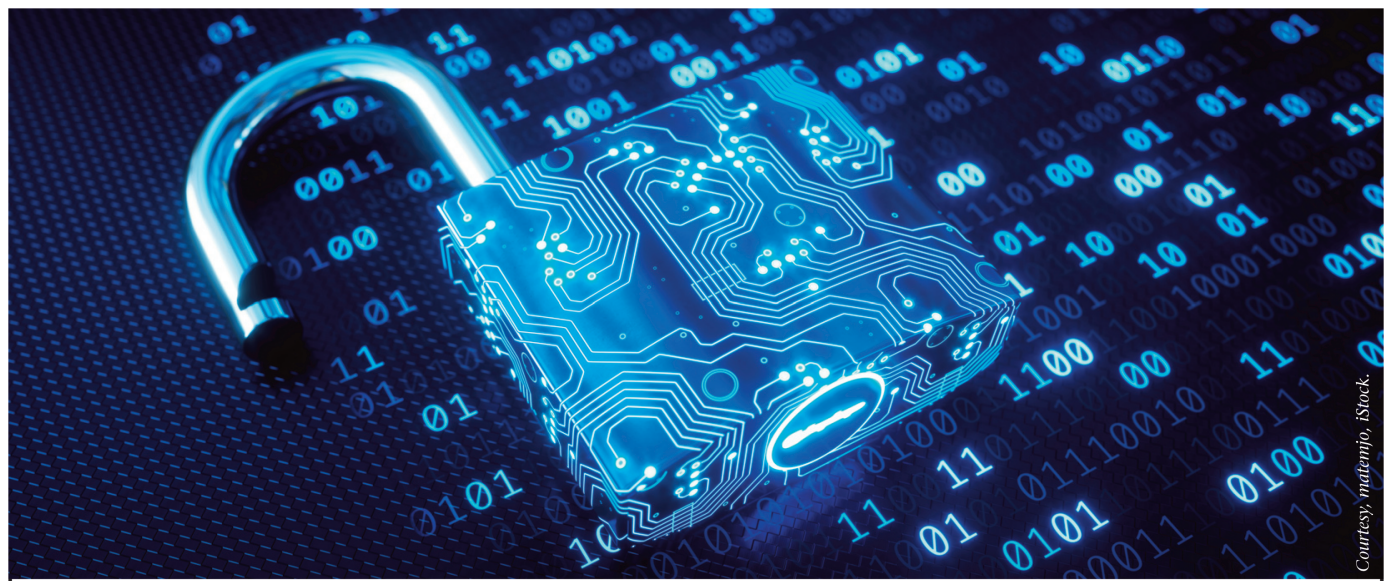

\section{Support AAAI Open Access}

AAAl counts on members like you to help us deliver the latest information about artificial intelligence to the scientific community. To enable us to continue this effort, we invite you to consider an additional gift to AAAl. For information on how you can contribute to the open access initiative, please click on "Gifts" at www.aaai.org, or select this option when renewing.

$A A A l$ is a $501 c 3$ charitable organization. Your contribution may be tax deductible. 